\title{
Global Stability Analysis and Stabilization of a class-E/F Amplifier With a Distributed Active Transformer
}

\author{
Sanggeun Jeon, Student Member, IEEE, Almudena Suárez, Senior Member, IEEE, and David B. Rutledge, Fellow, IEEE
}

\begin{abstract}
Power amplifiers (PAs) often exhibit instabilities giving rise to frequency divisions or spurious oscillations. The prediction of these instabilities requires a large-signal stability analysis of the circuit. In this paper, oscillations, hysteresis, and chaotic solutions, experimentally encountered in a high-efficiency class-E/ $F_{\text {odd }}$ PA with four transistors combined using a distributed active transformer, are studied through the use of stability and bifurcation analysis tools. The tools have enabled an in-depth comprehension of the different phenomena, which have been observed in simulation with good agreement with experimental results. The study of the mechanism generating the instability has led to a simplified equivalent circuit from which the optimum stabilization network has been determined. The network enables a global stabilization of the circuit for all the expected operating values of the amplifier bias voltage and input power. This has been achieved with negligible degradation of the amplifier performance in terms of drain efficiency and output power. The stable behavior obtained in simulation has been experimentally confirmed.
\end{abstract}

Index Terms-Bifurcation, chaos, distributed active transformer (DAT), hysteresis, stability analysis, stabilization, switching amplifiers.

\section{INTRODUCTION}

$\mathbf{S}$ WITCHING amplifiers such as classes D-F have been widely used to achieve high output power with high efficiency [1]. What distinguishes the different classes is the tuning technique of harmonics, the tolerance of transistor output capacitance, and the operating frequency limit. However, all of them are potentially able to achieve $100 \%$ drain efficiency by preventing the voltage and current waveforms from overlapping each other at a drain terminal. Since the transistor in switching amplifiers is operated as a switch instead of a current source to achieve high drain efficiency, the input-drive power should be large enough to make the transistor saturated for a certain time of period depending on a duty cycle.

A class-E/F amplifier has been proposed by Kee et al. [2] to combine the advantages of both class- $\mathrm{E}$ and class- $\mathrm{F}^{-1}$ operations. By selective tuning of harmonic components, the class-E/F amplifier can have the benefits of class $\mathrm{F}^{-1}$, which are low peak voltage and low rms current, using a simple

Manuscript received February 22, 2005; revised May 5, 2005. This work was supported by the Lee Center for Advanced Networking and by the Jet Propulsion Laboratory.

S. Jeon and D. B. Rutledge are with the Department of Electrical Engineering, California Institute of Technology, Pasadena, CA 91125 USA (e-mail: sjeon@ caltech.edu; rutledge@caltech.edu).

A. Suárez is with the Communications Engineering Department, University of Cantabria, 39005 Santander, Spain (e-mail: suareza@unican.es).

Digital Object Identifier 10.1109/TMTT.2005.856083 push-pull configuration. The class $\mathrm{E} / \mathrm{F}$ is also suitable at higher frequencies than the class $\mathrm{E}$ because it can tolerate a larger transistor output capacitance.

During the measurements of switching amplifiers, several different behaviors can be observed. Below a certain level of input power, the transistor is completely turned off and only leakage power from the input-drive source, passing through the feed-forward capacitance of the transistor, is obtained at the output. For an intermediate input power range, the output power and drain efficiency of the amplifiers increase rapidly. However, it is also not unusual to observe spurious oscillations, sub-harmonic oscillations, or even chaos when the amplifiers are not completely stable [3]-[5]. As the input drive increases further to a high power level, the amplifiers show a typical switching amplifier operation with high drain efficiency.

In switching amplifiers, the gain increase versus the input power for intermediate drive level, besides the negative resistance from nonlinear capacitances, may give rise to oscillations. These oscillations, observed from a certain level of input power, cannot be detected through a small-signal stability analysis of the circuit such as the one based on the $k$-factor and the stability circles. Instead, a large-signal stability analysis must be performed [6]-[8]. The qualitative changes in the observed spectrum, when varying the input power, are the result of bifurcations, or qualitative stability variations [9], taking place in the circuit.

In previous studies, large-signal analyses of the mechanisms leading single-ended or power-combining power amplifiers (PAs) to unstable behavior have been carried out [6]-[8], [10], [11]. As an example, the frequency division by two, commonly observed in power-combining amplifiers, has been related to odd-mode instabilities, favored by the symmetries of circuit topology. The analyzed amplifiers were operated in either classes A or AB. No similar study has ever been attempted in the case of switching amplifiers. To devise a proper stabilization procedure for these amplifiers, an understanding of the oscillation mechanism is necessary. This study will be carried out here through the use of accurate stability and bifurcation analysis tools, applied in combination with harmonic balance (HB). In particular, a PA at $29 \mathrm{MHz}$ exhibiting chaos and hysteresis will be analyzed in detail. The circuit contains two push-pull pairs with four vertical double-diffused MOSFETs (VDMOS) that are operated in class- $\mathrm{E} / \mathrm{F}_{\text {odd }}$ mode. The objective is to characterize the instability versus the bias voltage and input power. The instability contour, in terms of these two parameters, will be obtained through a bifurcation-detection technique. 


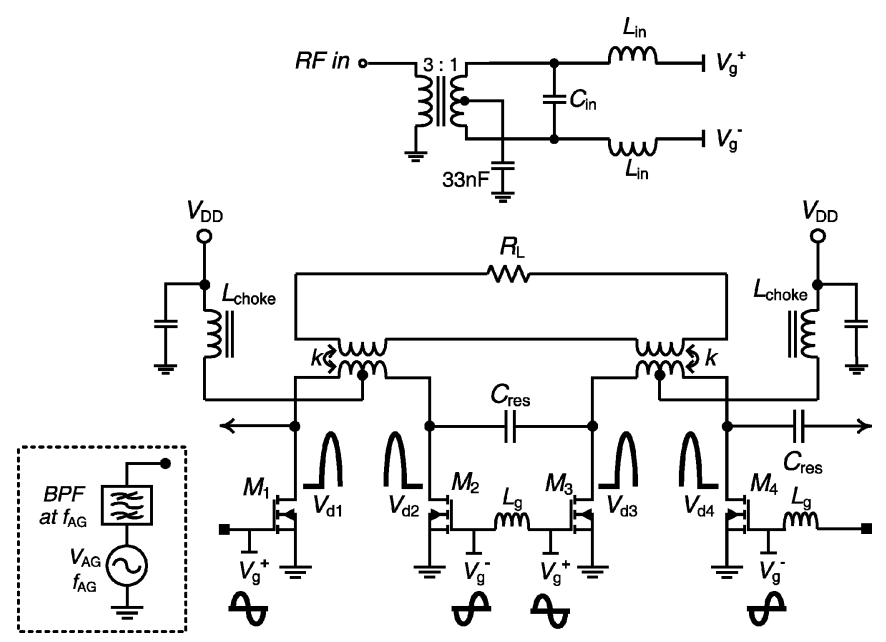

Fig. 1. Schematic of the class-E/Fodd $\mathrm{PA}$ with sketches of the voltage waveforms at the main circuit nodes. Two push-pull pairs are formed by $M_{1}-M_{2}$ and $M_{3}-M_{4}$, although the transistors in each pair are in different transistor packages. The AG with an ideal bandpass filter inside the dashed box is not a part of the amplifier, but will be connected to one of the drain terminals for the stability analysis in Section V.

A suitable stabilization network will then be designed in order to globally suppress the instability.

This paper is organized as follows. In Section II, the class-E/ $\mathrm{F}_{\text {odd }}$ PA operation is briefly summarized. In Section III, the measurement results, showing unstable behavior, are presented. In Section IV, the transistor-modeling efforts, in order to obtain reliable simulation results, are discussed. In Section V, the application of the stability and bifurcation analysis techniques is presented. Finally, Sections VI and VII, respectively, deal with the stabilization procedure and the measurement results.

\section{Operation of Class-E/F $F_{\text {odd }}$ PA With a Distributed ACTIVE TRANSFORMER (DAT)}

A class-E $/ \mathrm{F}_{\text {odd }} \mathrm{PA}$ has been designed and implemented with discrete components at $29 \mathrm{MHz}$. Four VDMOS transistors are combined using a distributed active transformer (DAT) [12], [13]. Fig. 1 shows a schematic of the amplifier with sketches of voltage waveforms at the gate and drain terminals. By the symmetry and double differential drive of the amplifier, each transistor pair $\left(M_{1}-M_{2}\right.$ and $\left.M_{3}-M_{4}\right)$ can be considered to be operated in the class-E $/ \mathrm{F}_{\text {odd }}$ mode independently. A capacitor $C_{\text {res }}$ and the magnetization inductance of the output transformer build a parallel resonance at the operating frequency, and present appropriate tuning impedances to the transistors at the fundamental and odd harmonics for the class-E/F $F_{\text {odd }}$ operation. Since each transistor pair is driven in push-pull, as shown in Fig. 1, a virtual ground develops at the center of the pair so that the impedance seen by a transistor is near zero at all odd harmonics if the $Q$ factor of the parallel resonant tank is high enough. Therefore, the drain voltage waveforms become half-sinusoidal and $180^{\circ}$ out-of-phase between each pair of transistors.

The secondary circuits of the two output transformers are connected in series, which forms a DAT. The DAT provides each transistor pair with a $2: 1$ output impedance transformation, as well as output power combining. The DAT is made of two pieces of thick copper tape, stacked together, with a cross section of

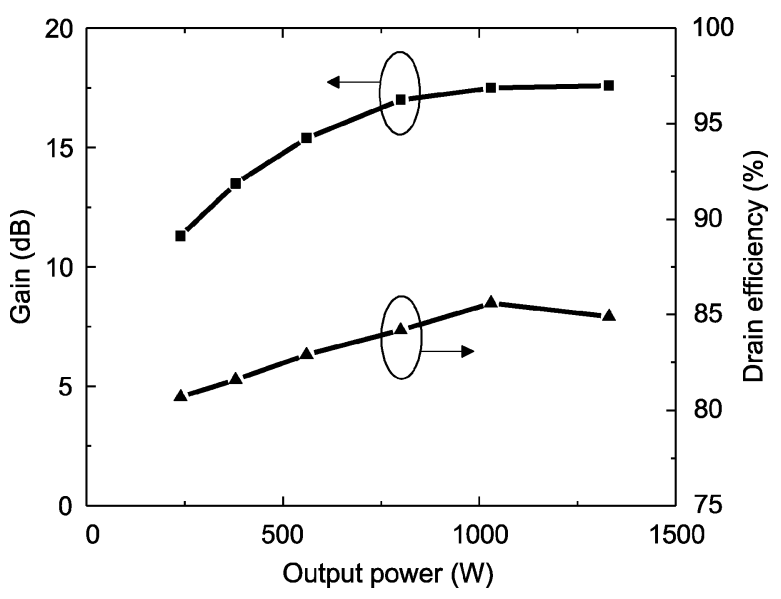

Fig. 2. Variation of the gain and drain efficiency of the class-E/ $F_{\text {odd }}$ PA versus the saturated output power. The output power is varied by changing the drain bias voltage.

$4.8 \mathrm{~mm} \times 1.3 \mathrm{~mm}$ each. The copper tape provides an inductance required for the output resonant tank with a high- $Q$ factor of 600 , which minimizes the ohmic loss under a high current flowing through them. It should be noted that the center of the primary circuit of the DAT is an ideal point for the dc feed owing to the virtual ground formed at the fundamental and odd harmonics.

\section{EXPERIMENTAL RESULTS}

The amplifier was experimentally characterized using a Yaesu FT-840 transceiver, a Bird oil-filled 30-dB 2-kW attenuator as a load, a Bird 4421 power meter, and an Agilent E4407B spectrum analyzer. Fig. 2 shows the measured gain and drain efficiency as a function of output power when the amplifier is driven by sufficiently large input power.

The variation of the measured output spectrum at a drain bias $V_{D D}=72 \mathrm{~V}$ with different input-drive power is presented in Fig. 3. As shown in Fig. 3(a), for low input power, only a leakage signal is obtained at the amplifier output. When the input power $P_{\text {in }}$ reaches $P_{\text {in_1 }}=5.5 \mathrm{~W}$, the output spectrum turns into the one in Fig. 3(b). The continuity of this spectrum suggests chaotic behavior. Two peaks can be seen on each side of the fundamental line at the input frequency of $29 \mathrm{MHz}$. The distance from each peak to this fundamental line is approximately $4 \mathrm{MHz}$. As the input power is further increased, this kind of spectrum continues to be observed until the input power reaches $P_{\text {in } \_2}=13.0 \mathrm{~W}$. From this value on, the spectrum becomes the proper one, shown in Fig. 3(c). The amplifier behaves in the expected switching mode with high drain efficiency (Fig. 2). Note that even harmonics are much more attenuated than odd harmonics because of the push-pull operation of the amplifier.

The reverse sense of input power variation has also been considered and we have found a hysteresis phenomenon. If the input power is reduced, the chaotic spectrum is observed until the value $P_{\text {in_3 }}=5.3 \mathrm{~W}$ is reached and a mixer-like spectrum is obtained. Thus, chaos is observed for the input power below the value $P_{\text {in_1 }}=5.5 \mathrm{~W}$ at which it had originated when increasing the power. As we decrease the power further from $P_{\text {in_3 }}=5.3 \mathrm{~W}$, the amplifier behaves in a self-oscillating mixer regime. The input signal at $29 \mathrm{MHz}\left(f_{\text {in }}\right)$ mixes with a self-oscillation at approximately $4 \mathrm{MHz}\left(f_{a}\right)$ and gives the output power 


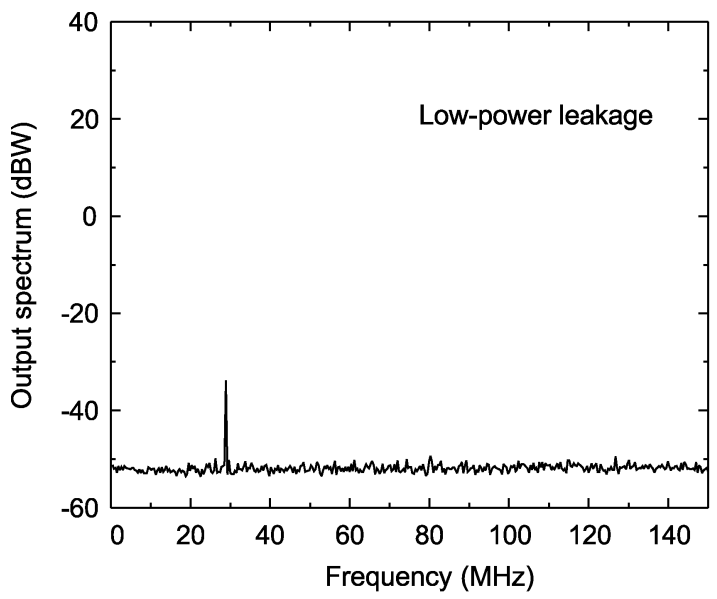

(a)

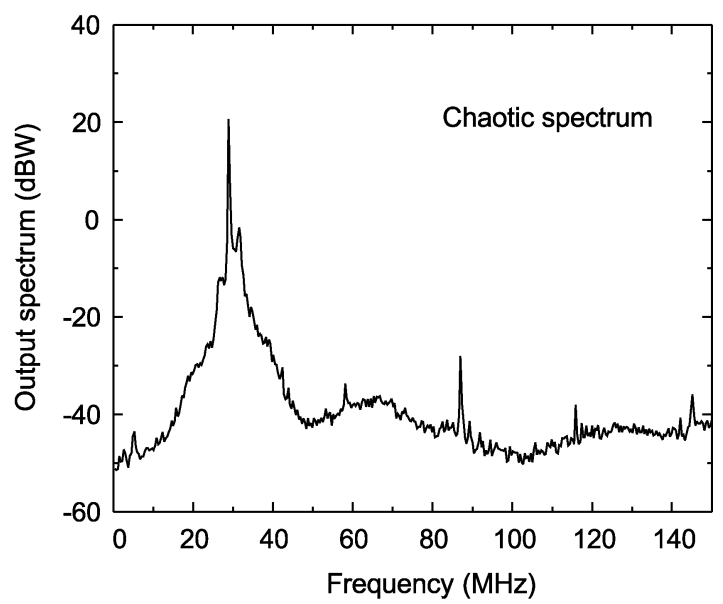

(b)

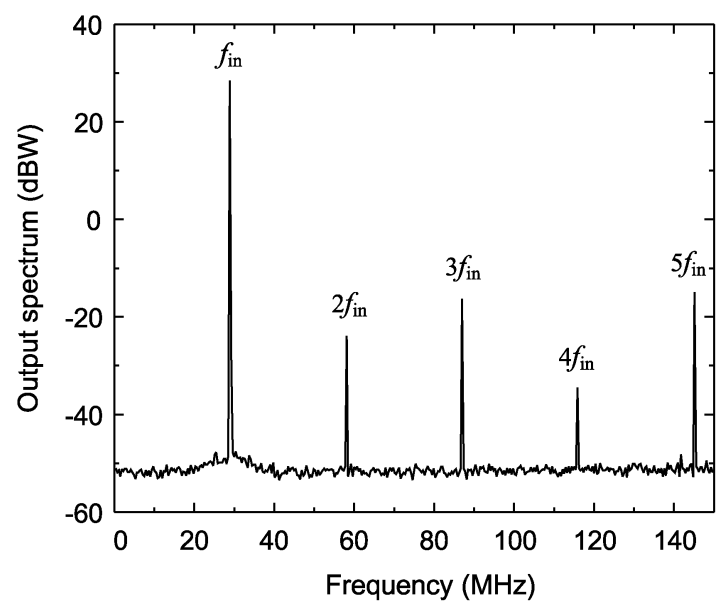

(c)

Fig. 3. Variation of the measured output power spectrum when increasing the input power. (a) $P_{\text {in }}=4 \mathrm{~W}$, showing leakage power at the input-drive frequency. (b) $P_{\text {in }}=10 \mathrm{~W}$, showing a chaotic spectrum. (c) $P_{\text {in }}=16.5 \mathrm{~W}$, showing the proper spectrum in switching-mode operation.

spectrum of Fig. 4. It is interesting to note that the intermodulation products with even orders at the oscillation frequency $f_{\text {in }} \pm 2 n f_{a}$ ( $n$ : positive integer) are stronger than those with odd orders at this frequency $f_{\text {in }} \pm(2 n-1) f_{a}$. This is attributed to the common-mode oscillation in each push-pull pair, which will be discussed in more detail in Section V-C. If the input power con-

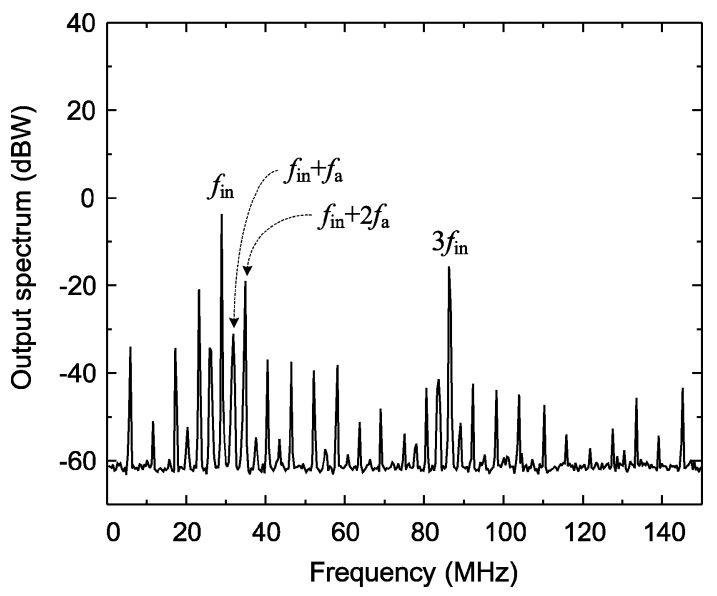

Fig. 4. Quasi-periodic output power spectrum observed near the bifurcation boundary when the input power is decreased. The circuit behaves in a selfoscillating mixer regime at the input-drive frequency $f_{\text {in }}=29 \mathrm{MHz}$ and the oscillation frequency $f_{a} \approx 4 \mathrm{MHz}$.

tinues to be reduced, the oscillation vanishes at the input power $P_{\text {in_4 }}=5.0 \mathrm{~W}$.

To summarize, as the input power increases, the amplifier undergoes bifurcations at the values $P_{\text {in_1 }}=5.5 \mathrm{~W}$ (jump to chaotic solution) and $P_{\text {in } \_2}=13.0 \mathrm{~W}$ (extinction of oscillation). When the input power decreases, the amplifier undergoes bifurcations at the values $P_{\text {in } \_3}=5.3 \mathrm{~W}$ (extinction of chaotic solution) and $P_{\text {in_4 }}=5.0 \mathrm{~W}$ (extinction of oscillation).

\section{TRANSISTOR MODELING}

As stated in Section I, one of the objectives of this study is to fully understand the different instability phenomena, involving self-oscillation, chaos, and hysteresis, which have been observed in the measurements. With this aim, stability and bifurcation analysis tools will be applied to the PA in combination with HB. In order for the simulation tools to be successful, accurate models for the different linear and nonlinear elements will be necessary. Thus, special effort has been devoted to the transistor modeling.

The active device employed in the amplifier is the ARF473 VDMOS from Advanced Power Technology Inc., Bend, OR [14]. It is a matched pair of power transistors with a maximum drain voltage of $500 \mathrm{~V}$ and rms drain current of $10 \mathrm{~A}$ for each transistor.

The transistor is modeled primarily as a voltage-controlled current source with two nonlinear capacitances [15], [16]. One is the drain-to-source capacitance $C_{\mathrm{ds}}$. This is modeled as a reverse-biased diode in which the parameters of the junction capacitance are fitted in order to match the measured capacitance as a function of the drain bias voltage. The other is the feedback capacitance between the gate and drain. The values of the feedback capacitance are extracted from the data sheet of the transistor, and a junction capacitance model is also used to fit the values. The gate-to-source capacitance is assumed to be constant as a first-order approximation [16]. The parasitic resistances and inductances at both gate and drain are also incorporated in the model as linear elements. 


\section{Stability AnAlysis}

As shown in Section III, different instability phenomena have been observed in the experimental characterization of the class-E $/ \mathrm{F}_{\text {odd }}$ amplifier including self-oscillation, chaos, and hysteresis. Thus, the stability analysis of the amplifier will be a demanding one, involving different kinds of tools. The entire analysis procedure is presented as follows.

\section{A. Local Stability Analysis}

The initial objective is to analyze the stability of the amplifier solution for several values of drain bias voltage and input power at which unstable behavior had been experimentally observed. The stability analysis is based on the linearization of the amplifier circuit about its large-signal steady-state regime at the input-drive frequency $f_{\text {in }}$ calculated with $\mathrm{HB}$ and 15 harmonic components. To obtain this linearization, a small-signal current generator is connected to a particular circuit node. Due to the complex topology of the amplifier circuit, different observation nodes must be considered. The generator operates at a frequency $f$, nonrationally related to $f_{\text {in }}$. The purpose of the generator is to enable the determination of the total impedance function $Z_{\text {in }}$ at the frequency $f$ at the observation node [17]. This is obtained by taking the ratio of the node voltage to the injected current using the conversion-matrix approach [18]. In this way, a single-input single-output transfer function is calculated to which pole-zero identification will be later applied.

Initially, the operation conditions of $V_{D D}=72 \mathrm{~V}$ and $P_{\text {in }}=$ $10 \mathrm{~W}$, for which instability had experimentally been observed, were considered. By sweeping $f$ and representing the real and imaginary parts of the admittance function $Y_{\text {in }}=1 / Z_{\text {in }}$, a critical resonance at $5.6 \mathrm{MHz}$ was found at the drain terminal of any of the four transistors. Negative conductance and a zero crossing of the susceptance with positive slope were obtained, which are the startup conditions for an oscillation at that frequency [19].

For a more rigorous stability analysis, pole-zero identification was applied to $Z_{\text {in }}$ [18]. Since all circuit nodes share the same characteristic equation [20], the pole values are independent of the particular location of the current generator. However, exact pole-zero cancellations may occur at some current-generator locations. Thus, the need for the initial consideration of different observation nodes arises. Applying this technique, a pair of complex-conjugate poles are found on the right-hand side of the complex plane for the considered conditions of $V_{D D}=72 \mathrm{~V}$ and $P_{\text {in }}=10 \mathrm{~W}$, confirming the unstable behavior (Fig. 5).

Now the variation of the input power will be considered. When increasing the input power from a very small value, the critical poles evolve, as shown in Fig. 5. The amplifier solution is initially stable with the poles located on the left-hand side of the complex plane. When increasing the input power, the critical poles cross the imaginary axis at $P_{\mathrm{in}_{-} H l}=6.1 \mathrm{~W}$. From this power value, the amplifier periodic solution becomes unstable. At $P_{\text {in_Hl }}$, a Hopf bifurcation [21] is obtained (the additional sub-index $l$ means lower boundary). This Hopf bifurcation gives rise to the onset of an oscillation at the frequency $4.8 \mathrm{MHz}$, which is determined by the imaginary part of the poles. As the power continues to increase, the poles move further to the right, turn, and cross the imaginary axis again to the left-hand side at

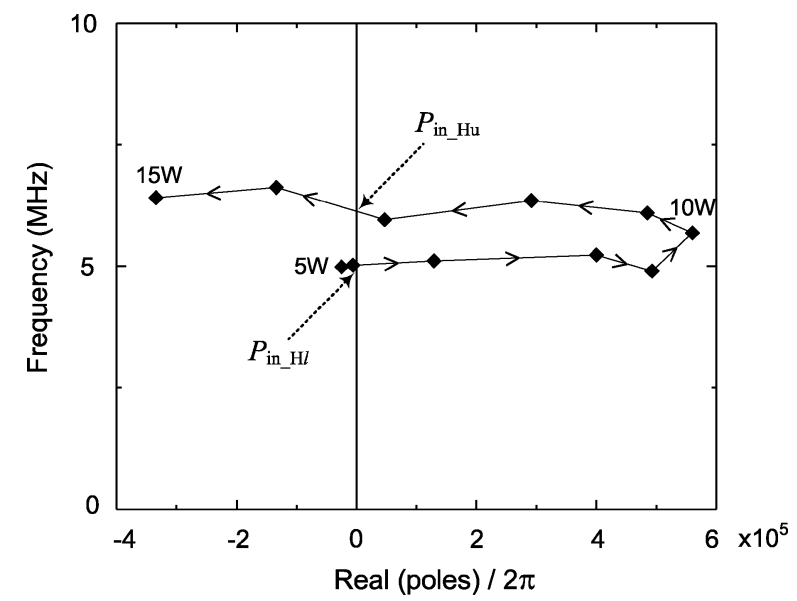

Fig. 5. Evolution of the critical poles with increasing input power for $V_{D D}=$ 72 V. For simplicity, only poles in the upper half of the complex plane have been represented. The input power has been increased from 5 to $15 \mathrm{~W}$ by $1-\mathrm{W}$ step. The poles cross to the right-hand side of the complex plane for $P_{\text {in_ } H l}=6.1 \mathrm{~W}$ and return to the left-hand side for $P_{\text {in_Hu }}=13.5 \mathrm{~W}$.

the input power value $P_{\text {in_Hu }}=13.5 \mathrm{~W}$ (the sub-index $u$ denotes upper boundary). At this power value, the oscillation vanishes. This corresponds to an inverse Hopf bifurcation occurring at $P_{\text {in_Hu }}$.

The above stability analysis provides the input-power range for which the amplifier periodic solution is unstable and, thus, unobservable. At $P_{\mathrm{in}_{-} H l}$, an oscillation is generated giving rise to a self-oscillating mixer regime. At $P_{\text {in } \_H u}$, the oscillation is extinguished and the amplifier recovers stability. Note that the stability analysis of the amplifier periodic solution does not enable by itself the prediction of the experimentally observed hysteresis phenomenon. Actually, in the experiment, chaotic and mixer-like spectra had been found for the input power below $P_{\text {in_Hl }} \mathrm{H}$ as well, which is not explained by the previous analysis. Hysteresis is associated with Hopf bifurcations of the subcritical type [21]. The determination of the bifurcation type requires higher order derivatives of the circuit equations about the bifurcation point [22], which is beyond the scope of this paper. A different technique will be used in this study, which will be demonstrated in Section V-D.

\section{B. Instability Contour}

The amplifier circuits generally have one or more parameters, susceptible to be varied in the different applications. The designer will be interested in knowing the parameter ranges, which give rise to unstable operation of the amplifier. In the case of this amplifier, the parameters are the drain bias voltage $V_{D D}$ and the input power $P_{\text {in }}$. Thus, the objective will be the determination of the set of $\left(V_{D D}, P_{\text {in }}\right)$ values with unstable behavior. The set will be delimited by the Hopf-bifurcation locus, containing the points at which the oscillation is generated or extinguished, depending on the variation sense of the parameters [21].

To obtain this locus, the continuity of local bifurcations is taken into account, according to which the oscillation amplitude tends to zero at the Hopf bifurcation. Unlike previous study [7], the small-signal current generator, introduced in Section V-A, will be used here to obtain the input-admittance function $Y_{\text {in }}$ at the observation node. This generator operates at a frequency 


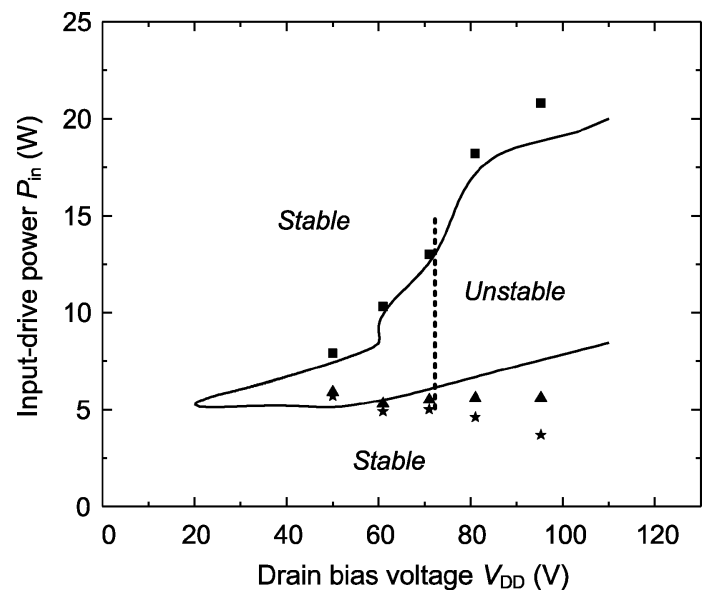

Fig. 6. Instability contour (solid line). The Hopf-bifurcation locus delimits the $V_{D D}$ and $P_{\text {in }}$ values for which the amplifier periodic solution is unstable. Experimental points have been superimposed. Squares indicate the upper border. In the lower border, triangles indicate the onset of instability for increasing input power, whereas stars indicate recovering of stable behavior for decreasing power. The dashed line shows the input power variation in the pole-zero identification of Fig. 5. Note that both analyses show good consistency in bifurcation points.

$f$, nonrationally related with $f_{\text {in }}$. The admittance $Y_{\text {in }}$ is calculated as the ratio between the delivered current and node voltage by means of the conversion-matrix approach. At the bifurcation point, occurring for $f=f_{a}$, both the real and imaginary parts of the input admittance vanish. This oscillation condition is fulfilled for oscillation amplitude tending to zero, as expected at the Hopf-bifurcation point. Thus, the Hopf-bifurcation locus, which delimits the unstable behavior region in terms of $V_{D D}$ and $P_{\text {in }}$, is obtained by solving the following system:

$$
Y_{\text {in }}\left(f_{a}, V_{D D}, P_{\text {in }}\right)=0
$$

with $f_{a}$ being the oscillation frequency. The above system (1) is solved through error minimization or optimization, using HB with 15 harmonic components and the conversion-matrix approach. The goals are $\operatorname{Real}\left(Y_{\text {in }}\right)=10^{-18} \mathrm{~S}$ and $\operatorname{Imag}\left(Y_{\text {in }}\right)=$ $10^{-18} \mathrm{~S}$. There exist three unknowns, i.e., $f_{a}, V_{D D}$, and $P_{\text {in }}$, in the two equations given by the real and imaginary parts of $Y_{\text {in }}$. This gives a curve in the plane defined by the bias voltage $V_{D D}$ and the input power $P_{\text {in }}$. Note that the oscillation frequency $f_{a}$ must be included in the calculation, as the frequency is autonomous and, thus, varies along the locus.

The application of the above technique to the class- $\mathrm{E} / \mathrm{F}_{\text {odd }}$ PA has provided the instability contour of Fig. 6. The dashed line shows the input power variation at $V_{D D}=72 \mathrm{~V}$ considered in the pole-zero identification of Fig. 5. The consistency in the bifurcation points resulting from both analyses should be noted. Through several applications of the pole-zero identification technique, the unstable region is confirmed to be inside the locus. The locus exhibits three points of infinite slope. To pass through these points, we switch the sweep parameter between $V_{D D}$ and $P_{\text {in }}$. Thus, the entire contour has been traced, enabling accuracy in the determination of the unstable operation region.

In Fig. 6, experimental points have been superimposed. In the lower border, two different sets of experimental points are represented. The triangles correspond to the points at which the amplifier becomes unstable for increasing input power. A chaotic regime is immediately obtained at most of the represented points, as shown in the spectra of Fig. 3. The stars correspond to the oscillation extinction for decreasing input power. The two sets of points show the hysteresis phenomenon discussed in Section III. On the other hand, in the upper border, no hysteresis has been experimentally obtained and only one set of measured points has been represented by squares. This set of points shows good agreement with the upper section of the simulated locus.

The instability contour provides the set of points at which the amplifier periodic solution becomes unstable, i.e., at which a pair of complex-conjugate poles cross the imaginary axis to the right-hand side of the complex plane. Thus, in the lower border, the contour must agree with the set of experimental points providing the instability threshold for increasing input power. As can be seen in Fig. 6, the obtained locus enables a good prediction of this set of values represented by triangles. The prediction of the hysteresis interval demands a different procedure to be presented in Section V-D.

\section{Analysis of the Self-Oscillating Mixer Regime}

For understanding of the oscillation mechanism, a steadystate analysis of the circuit in its undesired self-oscillating mixer regime has been carried out. The oscillating solution will exist inside the instability contour of Fig. 6. Due to the hysteresis phenomenon, it may also exist for input-power values below the lower border of the instability contour.

In order to obtain the oscillating solution in HB, a two-tone analysis must be carried out. One of the fundamentals is the input-drive frequency $f_{\text {in }}$. The other fundamental is the oscillation frequency $f_{a}$. By default, HB will converge to the amplifier periodic solution, with zeroes at all spectral lines containing $f_{a}$. In order to avoid this, an auxiliary generator (AG) is introduced into the circuit [21] for simulation purposes only. When choosing a voltage AG, this generator is connected in parallel at a circuit node. We will use the drain node, as in Section V-A. The AG operates at the oscillation frequency, i.e., $f_{\mathrm{AG}} \equiv f_{a}$, and must be an open circuit at all other frequencies. Thus, an ideal bandpass filter is used in series with the AG (Fig. 1). Furthermore, the AG must not perturb the circuit steady-state solution. This is ensured by imposing a zero value to its current-to-voltage relationship $Y_{\mathrm{AG}}=I_{\mathrm{AG}} / V_{\mathrm{AG}}=0$, where $I_{\mathrm{AG}}$ and $V_{\mathrm{AG}}$ are the current and voltage of the AG, respectively. For given $V_{D D}$ and $P_{\text {in }}$, the amplitude $V_{\mathrm{AG}}$ and the frequency $f_{\mathrm{AG}}$ of the $\mathrm{AG}$ are calculated in order to fulfill the condition $Y_{\mathrm{AG}}\left(V_{\mathrm{AG}}, f_{\mathrm{AG}}\right)=0$. Even though the amplifier contains four transistors, only one $\mathrm{AG}$, connected at one of the drain terminals, is necessary to determine the oscillating steady state.

To investigate the nature of the oscillation, the phase at each drain terminal of the four transistors has been analyzed at different harmonic frequencies (see Table I). At the oscillation frequency $f_{a}$, the two transistors in the same pair are in-phase, whereas the two pairs are $180^{\circ}$ out-of-phase. However, at the input-drive frequency $f_{\text {in }}$, the original phase-shift relationships are maintained, i.e., $180^{\circ}$ phase shift between the two transistors in the same pair and $180^{\circ}$ phase shift between the two pairs as well. Other phase relationships exist at intermodulation products of the two frequencies. The oscillation can be understood 
TABLE I

Phase of Signals With DifFERENT FreQuencies at EACH Drain TERMinal OF THE Four TRANSISTORS $\left(V_{D 1} \sim V_{D 4}\right.$ ARE DEFINED IN FIG. 1$)$

\begin{tabular}{c|c|c|c|c}
\hline Frequency & $V_{\mathrm{d} 1}$ & $V_{\mathrm{d} 2}$ & $V_{\mathrm{d} 3}$ & $V_{\mathrm{d} 4}$ \\
\hline$f_{\mathrm{a}}$ & $0^{\circ}$ & $0^{\circ}$ & $180^{\circ}$ & $180^{\circ}$ \\
\hline$f_{\text {in }}-f_{\mathrm{a}}$ & $156^{\circ}$ & $-64^{\circ}$ & $-64^{\circ}$ & $156^{\circ}$ \\
\hline$f_{\text {in }}$ & $-71^{\circ}$ & $109^{\circ}$ & $-71^{\circ}$ & $109^{\circ}$ \\
\hline$f_{\text {in }}+f_{\mathrm{a}}$ & $-86^{\circ}$ & $118^{\circ}$ & $118^{\circ}$ & $-86^{\circ}$ \\
\hline
\end{tabular}

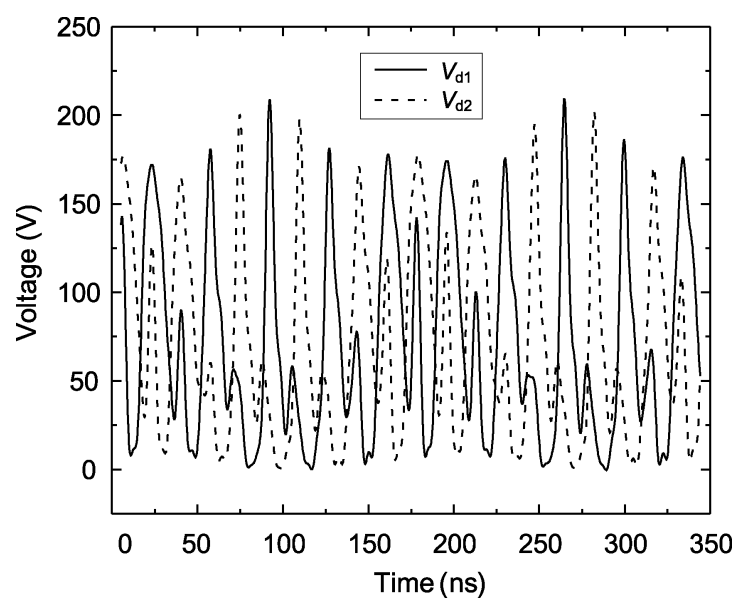

Fig. 7. Comparison of two simulated drain voltage waveforms $V_{d 1}$ and $V_{d 2}$ in the same push-pull pair. The slowly varying envelopes at the oscillation frequency appear to be in-phase, whereas the fast-varying carriers are out-of-phase.

as the result of the negative resistance exhibited by the transistor under relatively strong pumping signal and the resonant circuit formed with the equivalent capacitance and inductance seen from the drain terminals. This equivalent circuit will be discussed in detail in Section VI.

The two drain voltage waveforms in the same push-pull pair, i.e., $V_{d 1}$ and $V_{d 2}$, are compared in Fig. 7. It can be seen that the slowly-varying envelopes at $f_{a}$ are in-phase, whereas the fastvarying carriers at $f_{\text {in }}$ show a $180^{\circ}$ phase shift from each other.

Since the amplifier is operated in push-pull, the in-phase drain voltage waveforms at the oscillation frequency will ideally be cancelled, presenting no power in the output spectrum. This explains why the intermodulation products of the form $f_{\text {in }} \pm(2 n-1) f_{a}$ ( $n$ : positive integer) are much more attenuated than those having the form $f_{\text {in }} \pm 2 n f_{a}$, as can be seen in Fig. 4 . In a practical amplifier, however, no perfect cancellation can occur due to the imperfect symmetry.

Taking the above phase relationships into account, different virtual-ground and virtual-open planes can be considered in the circuit topology. At the input-drive frequency $f_{\text {in }}$, virtual-ground planes exist between any of two adjacent transistors. At the oscillation frequency $f_{a}$, two virtual-ground planes are located between the two push-pull pairs, as shown in Fig. 8. In addition, two virtual-open planes develop at the symmetry planes of the pairs.

Considering these virtual-ground and virtual-open plane concepts, a simplified equivalent circuit at the oscillation frequency will be obtained in Section VI, which will be useful in efficiently finding the stabilization network.

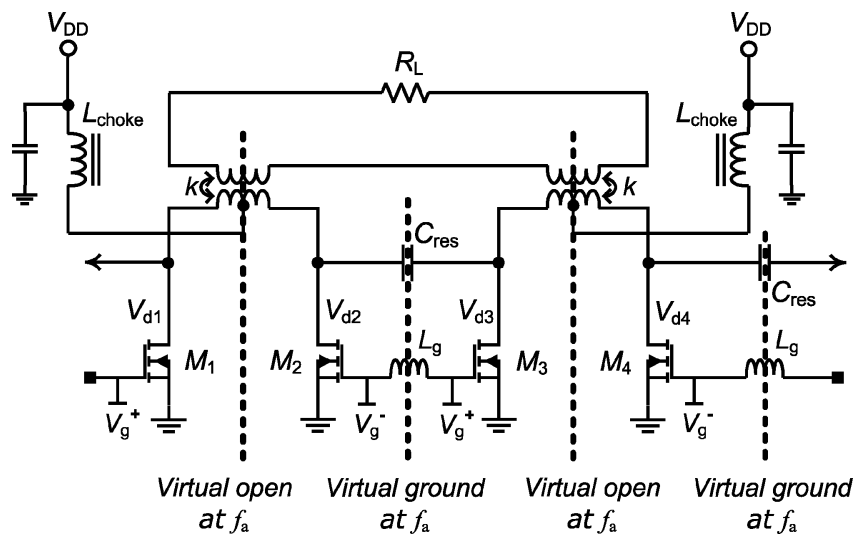

Fig. 8. Schematic of the push-pull amplifier showing the virtual-ground and virtual-open planes at the oscillation frequency.

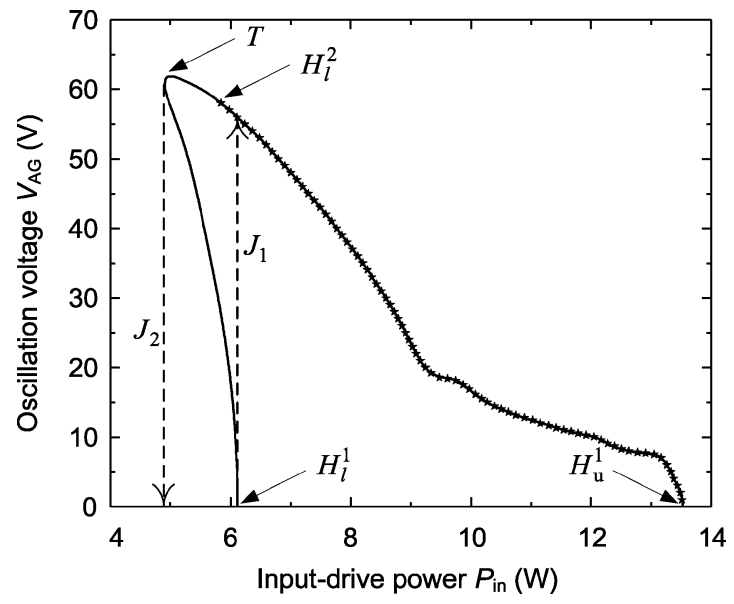

Fig. 9. Simulation of the undesired self-oscillating mixer regime of the PA. Variation of the oscillation amplitude at the drain terminal is represented with the input-drive power. The points at which the different bifurcations occur are indicated. $H$ denotes a Hopf bifurcation and $T$ denotes a turning point. $H_{l}^{1}$ and $H_{u}^{1}$ are Hopf bifurcations from the amplifier periodic regime. $H_{l}^{2}$ is a Hopf bifurcation from the self-oscillating mixer regime. $J_{1}$ and $J_{2}$ indicate jumps of the solution. Chaotic solutions are observed from $H_{l}^{2}$, which is analyzed in Section V-E.

\section{Hysteresis Prediction}

To study the hysteresis phenomenon, the evolution of the self-oscillating mixer solution versus the input power will be analyzed. As discussed in Section V-C, an AG is connected to any drain terminal and the equation $Y_{\mathrm{AG}}\left(V_{\mathrm{AG}}, f_{\mathrm{AG}}\right)=0$ is solved versus $P_{\text {in }}$ in combination with $\mathrm{HB}$. A two-fundamental frequency basis at $f_{\text {in }}$ and $f_{\mathrm{AG}}$, the latter playing the role of the oscillation frequency $f_{a}$, must be considered in the HB simulation. The resulting variation of the oscillation amplitude at the drain terminal, agreeing with $V_{\mathrm{AG}}$, is represented in Fig. 9. A constant bias voltage $V_{D D}=72 \mathrm{~V}$ has been assumed. As can be seen, the curve exhibits an infinite-slope point or turning point $T$. To pass through this point, the sweep parameter has been switched to the oscillation amplitude $V_{\mathrm{AG}}$ in the neighborhood of the turning point, calculating the input power $P_{\text {in }}$ and the oscillation frequency $f_{\mathrm{AG}}$ for each $V_{\mathrm{AG}}$ value.

The turning point is responsible for the hysteresis phenomenon. Actually, when the input power is increased, the transition from the stable amplifier behavior to the self-oscillating mixer regime ( $J_{1}$ in Fig. 9) is due to a Hopf bifurcation $H_{l}^{1}$ occurring 
in the amplifier solution. When the input power is decreased, the transition back to the stable amplifier behavior $\left(J_{2}\right)$ is due to the turning point $T$ in the self-oscillating mixer solution. Note that the simulated hysteresis interval, in terms of the input power, is in good correspondence with the experimental one shown in Fig. 6. On the other hand, no hysteresis is obtained in the upper input-power range, delimited by $H_{u}^{1}$, which also agrees with the measurement results.

The hysteresis phenomenon is well predicted by Fig. 9. However, in the measurement, an abrupt transition from stable amplifier behavior to the chaotic regime occurred for most $V_{D D}$ values, when the input power was increased. The study of this chaotic solution will require additional tools, to be presented in Section V-E.

\section{E. Envelope-Transient Analysis of the Oscillating Solution}

The envelope-transient enables an efficient analysis of the regimes in which two different time scales may be distinguished. In this technique, the circuit variables are expressed in a Fourier series with time-varying coefficients and a differential-equation system is obtained in these coefficients [23], [24]. The technique is efficiently applied to forced circuits. However, when used for the simulation of an oscillating regime, like that of the unstable amplifier, it generally converges to the coexisting nonoscillating solution in a similar manner to HB. To avoid this, the oscillation must be properly initialized [25]. This can be done through the connection of an AG to the circuit at the initial envelope time $t_{0}$. The amplitude and frequency of the AG are determined through a previous HB simulation. Since the AG is used for the initialization of the solution only, it must be disconnected from the circuit for time $t>t_{0}$. After this disconnection, the circuit will evolve according to its own dynamics. The AG disconnection from the circuit can be carried out with the aid of a time-varying resistor in series with the AG [25] changing from zero to a very high value (ideally infinite).

The objective will be to analyze the circuit along the entire solution curve of Fig. 9, corresponding to a self-oscillating mixer regime. Thus, the variables are represented here in a Fourier series with $f_{\text {in }}$ and $f_{\mathrm{AG}}$ as fundamentals, i.e., $\mathbf{x}(t)=\sum_{k, l} \mathbf{X}_{k, l}(t) e^{j 2 \pi\left(k f_{\text {in }}+l f_{\mathrm{AG}}\right) t}$. At each point, the AG amplitude $V_{\mathrm{AG}}$ and frequency $f_{\mathrm{AG}}$, resulting from the $\mathrm{HB}$ analysis in Fig. 9, are used for initialization purposes. From the point $H_{l}^{2}$ in Fig. 9, the magnitude of the harmonic components $\left|\mathbf{X}_{k, l}(t)\right|$ becomes time-varying [see Fig. 10(a)]. It oscillates at a few hundred kilohertz, the actual oscillation frequency depending on the input power. Thus, there is a second oscillation, in addition to the previous oscillation at approximately $4 \mathrm{MHz}$ $\left(f_{a}\right)$. Together with the input-drive frequency, this gives rise to a three-fundamental regime. The simulated spectrum is shown in Fig. 10(b). To verify this qualitatively, an expanded view of the experimental spectrum near the turning point $T$ in Fig. 9 is shown in Fig. 11. This confirms the existence of the second oscillation at approximately $500 \mathrm{kHz}$, in agreement with the envelope-transient simulation, which has enabled the efficient detection of the second oscillation.

As has been shown, there are two autonomously generated fundamentals involved in the circuit solution in addition to the input-drive frequency. According to the Ruelle-Takens theorem

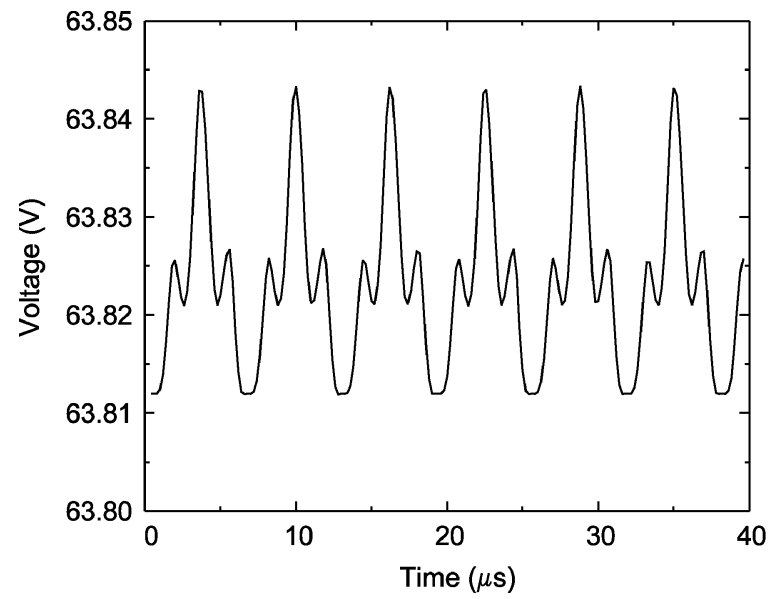

(a)

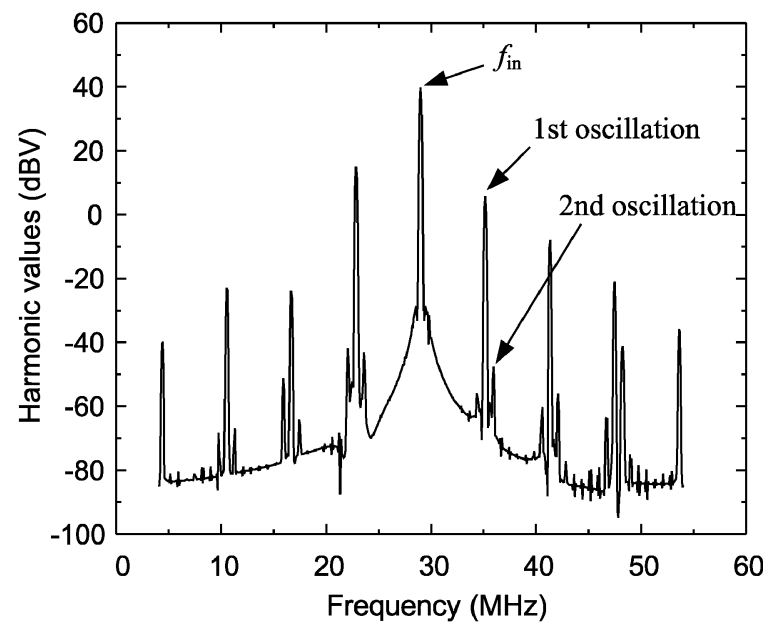

(b)

Fig. 10. Envelope-transient simulation of the amplifier. (a) Time-domain evolution of the magnitude of the $f_{\text {in }}$ harmonic component of the drain voltage when a two-fundamental basis at $f_{\text {in }}$ and $f_{a}$ is considered. (b) Spectrum of the harmonic component showing the presence of two oscillation frequencies.

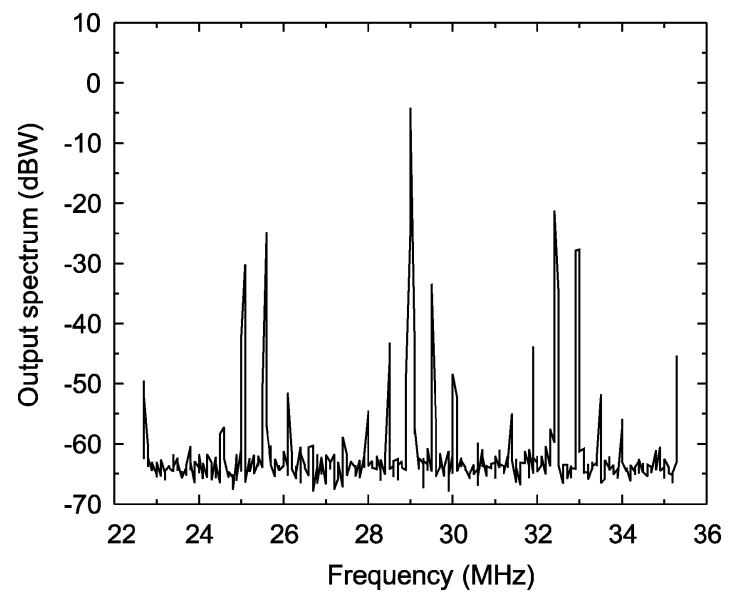

Fig. 11. Expanded view of the experimental output power spectrum about the input-drive frequency. $V_{D D}=72 \mathrm{~V}$ and $P_{\text {in }}=5.15 \mathrm{~W}$. It shows the coexistence of two oscillation frequencies at approximately $4 \mathrm{MHz}$ and $500 \mathrm{kHz}$. This is in agreement with the envelope-transient simulation of Fig. 10.

[9], this type of solution is likely to give rise to chaos, which would explain the chaotic spectrum that was observed in the experiment [see Fig. 3(b)]. Actually, chaotic envelope variations 
have also been obtained in simulation for some values of input power. However, it should be noted that there is limited accuracy in the representation of these solutions using the two-tone basis $f_{\text {in }}$ and $f_{\mathrm{AG}}$.

The interval in which the self-oscillating mixer solution at $f_{\text {in }}$ and $f_{a}$ is unstable has been indicated with stars in Fig. 9. At the point $H_{l}^{2}$, the self-oscillating mixer solution becomes unstable due to the generation of the second oscillation frequency. It is a Hopf bifurcation from the self-oscillating mixer regime. Thus, starting from very low input power, the amplifier periodic solution is initially stable, and as the input power is further increased, it suddenly becomes chaotic at the Hopf-bifurcation point $H_{l}^{1}$. This is because the input power for the bifurcation $H_{l}^{1}$ is larger than the input power for the bifurcation $H_{l}^{2}$ so the solution jumps from $H_{l}^{1}$ to the chaotic regime (see $J_{1}$ in Fig. 9). The chaotic regime persists until the input power reaches the bifurcation point $H_{u}^{1}$ from which the amplifier periodic solution becomes stable.

When decreasing the input power, the second oscillation vanishes at $H_{l}^{2}$ and the self-oscillating mixer regime (at $f_{\text {in }}$ and $f_{a}$ ) becomes stable for a very short input-power interval. At the turning point $T$, the system jumps to the stable amplifier periodic solution ( $J_{2}$ in Fig. 9).

In conclusion, the bifurcation diagram of Fig. 9 gives a satisfactory explanation of the experimental observations of Fig. 3. All the different phenomena observed in the measurements are associated with the occurrence of particular types of bifurcations.

\section{Stabilization Technique}

After understanding the different phenomena observed in the measurements, the objective will be the stabilization of the amplifier. For this purpose, a stabilization network will have to be added to the circuit. In order to efficiently obtain the optimum network, a simplified equivalent circuit will be derived here taking into account the virtual-ground and virtual-open planes at the oscillation frequency, identified in Section V-C.

Fig. 12 shows the equivalent circuit that corresponds to a quarter section of the amplifier at the oscillation frequency. It is a parallel resonance oscillator composed of the transistor exhibiting negative resistance and the equivalent capacitance, inductance, and load resistance seen from the drain terminal. The output capacitance $C_{\text {res }}$ connected between two transistor pairs is doubled due to the virtual-ground developed at the center of the capacitance, and the magnetization inductance $L_{\text {res }}$ in the output transformer is divided by two due to the virtual-open at the center of the transformer. Note that the RF choke inductance $L_{\text {choke }}$ is also a critical element included in the equivalent circuit. The resonance frequency is $5.3 \mathrm{MHz}$, which is quite close to the oscillation frequency obtained both in measurement and simulation. This confirms the validity of the proposed equivalent circuit.

From the schematic of Fig. 12, a simple means to stabilize the amplifier is the addition of a resistor at node $N$. The value of this resistance must be small enough to avoid the oscillation for all the possible operation conditions in terms of $V_{D D}$ and $P_{\text {in }}$. It also must not affect the normal operation of the amplifier.

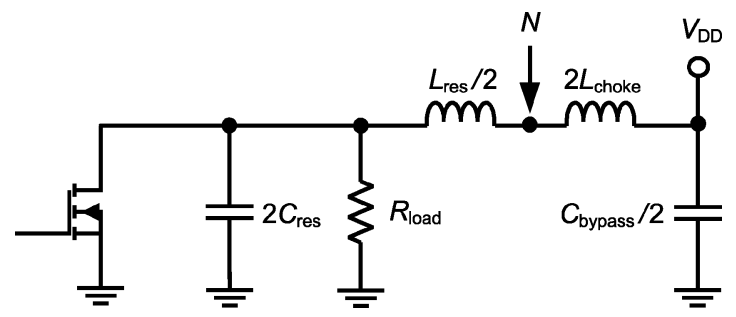

Fig. 12. Simplified equivalent circuit of the PA at the oscillation frequency after considering the virtual-open and virtual-ground planes.

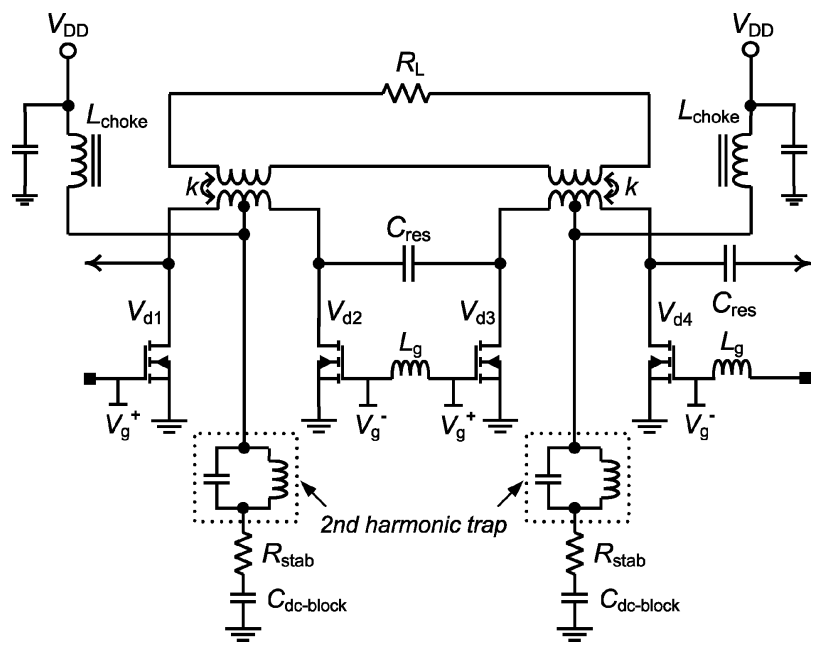

Fig. 13. Amplifier schematic with stabilization network. The stabilization network consists of a stabilization resistor $R_{\text {stab }}$, a second harmonic trap, and a dc-blocking capacitor $C_{\text {dc-block }}$.

The node $N$ corresponds to the center point of the primary circuit in the output transformers. The push-pull operation introduces a virtual ground at the node for the operating frequency $f_{\text {in }}$ and odd harmonics. Hence, the addition of the resistance at the node $N$ will have little effect over these frequencies. However, the resistor will impose a finite impedance to even harmonics instead of an open circuit, which would be the right termination for the class-E/ $\mathrm{F}_{\text {odd }}$ operation. Thus, a second harmonic trap at $2 f_{\text {in }}$ will be connected in series with the resistor to reduce the effect. This will provide an open circuit at the second harmonic frequency. The effect of higher even harmonics on the operation, expected to be small, will be analyzed through simulation. The schematic of the amplifier with the stabilization network is shown in Fig. 13.

Once the topology and location of the stabilization network have been determined, the next step will be the calculation of stabilization resistance value, in order to ensure stable amplifier operation for all the expected values of $V_{D D}$ and $P_{\text {in }}$. An efficient technique will be applied for this purpose. The technique is based on the plot of the small-signal input admittance $Y_{\text {in }}$. This is calculated at the drain terminal using the small-amplitude current source and the conversion-matrix approach (see Section V-A). Comparing the frequency variation of $Y_{\text {in }}$ with pole-zero identification results, it has been possible to associate the instability with the existence of negative conductance and resonance at the oscillation frequency. Thus, the plot of $Y_{\text {in }}$ will allow a fast verification of these oscillation conditions. 
Three different values of the stabilization resistance have been considered, i.e., 100,50 , and $15 \Omega$. For each value, two nested sweeps are carried out in the two amplifier parameters $V_{D D}$ and $P_{\text {in }}$. For each $\left(V_{D D}, P_{\text {in }}\right)$ point, an HB calculation is performed, together with a sweep in the current-source frequency $f$, using a conversion matrix. This yields the input admittance $Y_{\text {in }}(f)$ seen by the current source. The imaginary part of $Y_{\text {in }}(f)$ is then plotted versus the real part. The resulting plots for the original amplifier and amplifier with three indicated resistance values are shown in Fig. 14. Each admittance curve corresponds to a pair of values $\left(V_{D D}, P_{\text {in }}\right)$. The same frequency-sweep range has been considered for each curve. In the representation, this range has been limited to $3-5.5 \mathrm{MHz}$ for the sake of clarity. For global stability, no crossing of the real axis with negative conductance and positive increase of the susceptance must be obtained [19]. As expected, larger stability ranges are achieved as the stabilization resistance is reduced. For $R_{\text {stab }}=15 \Omega$, the amplifier becomes stable for all the operation values of $V_{D D}$ and $P_{\text {in }}$. This has been rigorously verified by extending the range of the frequency sweep and applying pole-zero identification.

Through bifurcation detection, it is possible to directly calculate the stabilization resistance $R_{\text {stab }}$ for given $V_{D D}$ and $P_{\text {in }}$ values. To achieve this, the stabilization resistance $R_{\text {stab }}$ and the oscillation frequency $f_{a}$ will be determined in order to fulfill $Y_{\text {in }}\left(R_{\text {stab }}, f_{a}\right)=0$. For each $V_{D D}$ and $P_{\text {in }}$, the resulting resistance $R_{\mathrm{stab}}^{0}$ is the maximum value allowed for stable behavior. The resistance value $R_{\mathrm{stab}}^{0}$ is actually a bifurcation value: the amplifier is unstable for $R_{\mathrm{stab}}>R_{\mathrm{stab}}^{0}$, whereas it is stable for $R_{\text {stab }}<R_{\text {stab }}^{0}$.

In order to globally determine the variation of $R_{\text {stab }}^{0}$, a sweep of $P_{\text {in }}$ is performed for several $V_{D D}$ values covering the expected operation ranges. For each $V_{D D}$, the equation $Y_{\text {in }}\left(R_{\text {stab }}, f_{a}\right)=0$ is solved to calculate $R_{\text {stab }}^{0}$ versus $P_{\text {in }}$, which is shown in Fig. 15. As can be observed, $R_{\text {stab }}^{0}$ decreases with the bias voltage. On the other hand, as $P_{\text {in }}$ approaches values for which the amplifier periodic solution is stable, this resistance tends to infinity. From Fig. 15, a resistance value smaller than $17 \Omega$ is required for global stabilization of the amplifier. The results are consistent with those obtained from the admittance plots of Fig. 14.

In view of the results of Figs. 14 and 15, the resistance value $R_{\text {stab }}=15 \Omega$ has been chosen for the corrected design of the amplifier. As the final step, the influence of this resistance on the amplifier drain efficiency and output power has been analyzed. This is shown in Fig. 16, where the drain efficiency and output power is traced versus the resistance value. As can be seen, the stabilization resistance has only a small influence. This is due to the fact that the connection point is a virtual ground at the fundamental and odd harmonics, and the second-harmonic trap has been used to maintain the connection point as an open circuit at that frequency. The higher even harmonics turned out to have negligible influence.

\section{MEASUREMENTS OF THE STABILIZED AMPLIFIER}

The amplifier has been modified for globally stable behavior by introducing the stabilization network developed in Section VI. A stabilization resistor of $15 \Omega$ in series with the

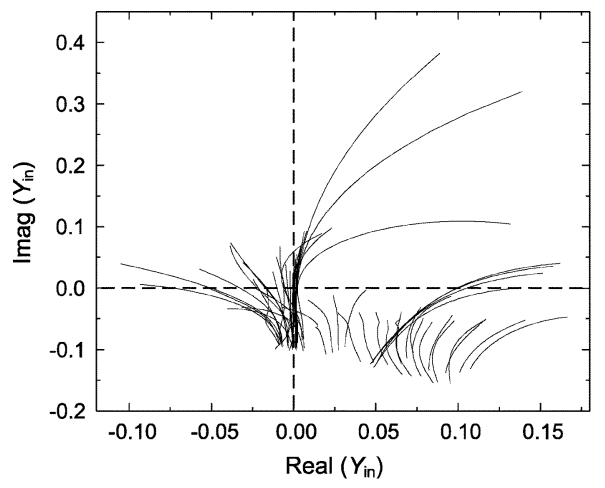

(a)

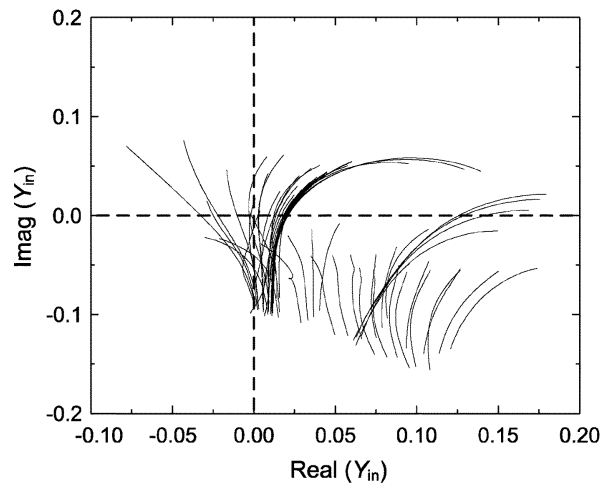

(b)

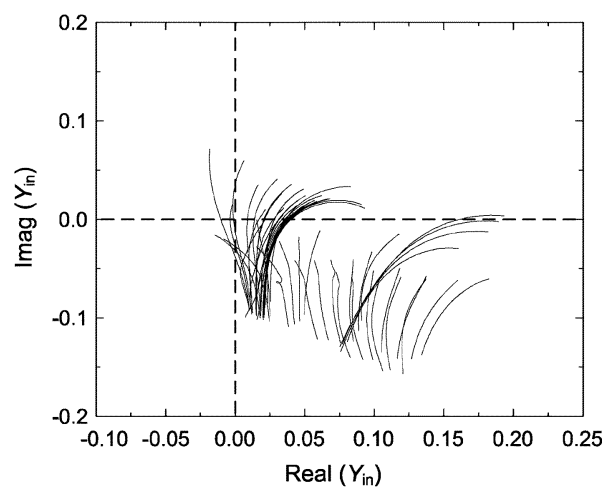

(c)

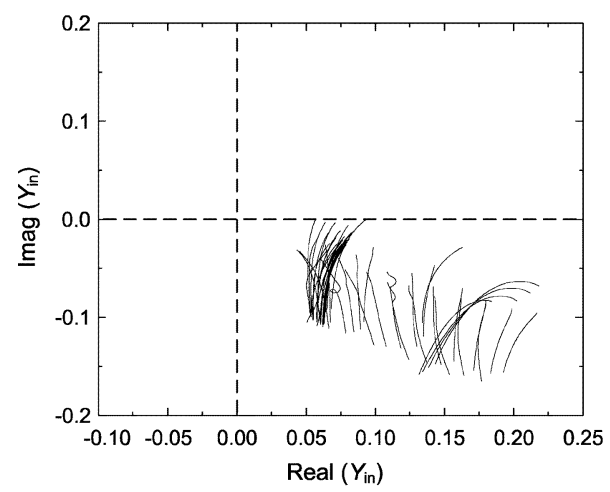

(d)

Fig. 14. Stabilization action of the parallel resistance analyzed by means of admittance plots. Three resistance values have been considered. (a) No stabilization resistor. (b) $R_{\text {stab }}=100 \Omega$. (c) $R_{\text {stab }}=50 \Omega$. (d) $R_{\text {stab }}=15 \Omega$. In (a)-(c), the oscillation condition is satisfied for a certain parameter range and, thus, global stability is not achieved.

second harmonic trap and a dc-blocking capacitor is simply connected to the center point of each output transformer. Power 


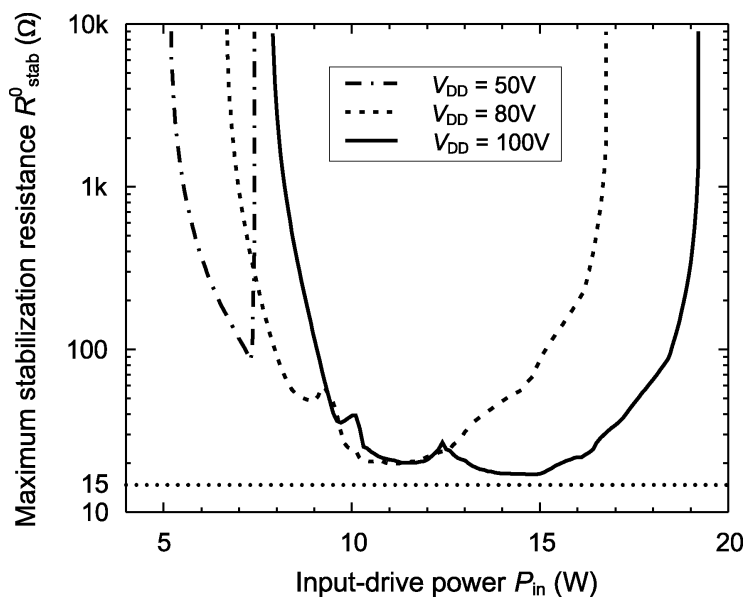

Fig. 15. Variation of the maximum value of stabilization resistance $R_{\text {stab }}^{0}$ versus the input-drive power $P_{\text {in }}$ obtained through bifurcation analysis. Three different drain bias voltages have been considered.

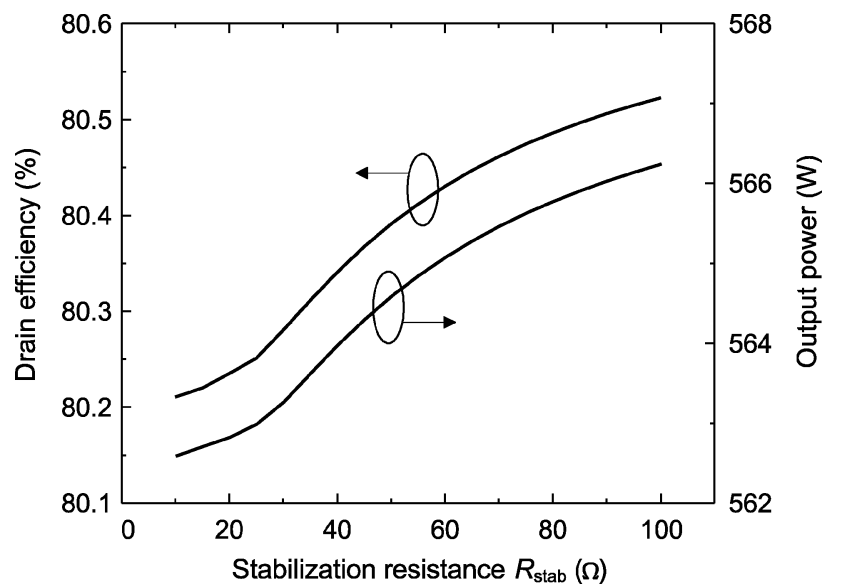

Fig. 16. Variation of the amplifier drain efficiency and output power versus the value of the stabilization resistance. The drain bias voltage is assumed as $V_{D D}=72 \mathrm{~V}$.

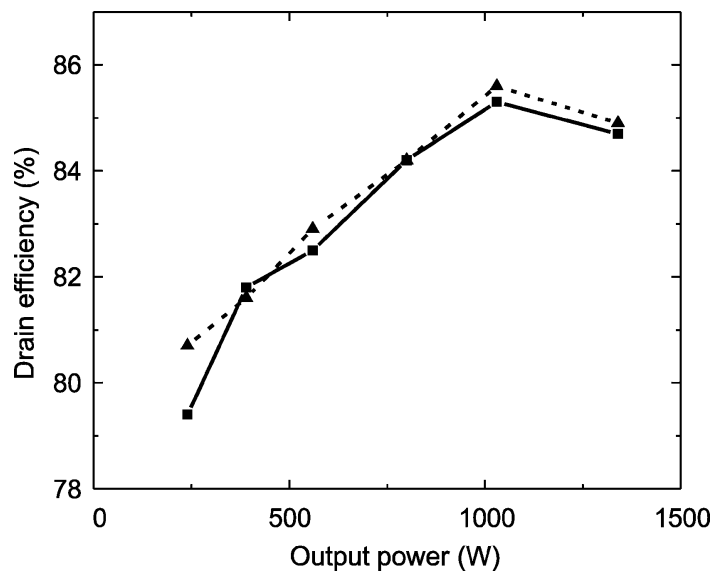

Fig. 17. Measured drain efficiency versus the output power. Solid line: stabilized PA. Dashed line: original PA. Compared to the original PA, the drain efficiency is degraded by less than $0.4 \%$ for all output power levels, except for $240 \mathrm{~W}$, which shows $1.3 \%$ degradation.

resistors with a $35-\mathrm{W}$ rating are used in order to handle the simulated current of $0.7 \mathrm{~A}$ at even harmonics of the input-drive frequency. The $Q$ factor of the second harmonic trap is carefully chosen considering a tradeoff between low impedance at the oscillation frequency and the feasibility of realizing each component. A mica capacitor and an air-core inductor with 14-AWG copper wire are used for the second harmonic trap.

This amplifier has shown global stability over the entire range of operating conditions of $V_{D D}$ and $P_{\text {in }}$, as predicted in the simulation. We never observed any oscillation or chaotic regimes. The output power spectrum was similar to Fig. 3(c) whenever the input drive was sufficient to turn on the transistors. The drain efficiency of the amplifier has been measured, which is shown in Fig. 17. As can be seen, the only significant degradation is $1.3 \%$ for $240-\mathrm{W}$ output power.

\section{CONCLUSION}

In this study, the unstable behavior of a class-E/F PA, experimentally exhibiting self-oscillation, chaos, and hysteresis, has been studied. Stability and bifurcation analysis tools have been applied to give satisfactory explanation to the different phenomena observed in the experiment, which are associated with particular kinds of bifurcations. An in-depth analysis of the oscillation mechanism has also been carried out. From this study, a simplified equivalent circuit at the oscillation frequency has been derived, enabling an efficient determination of the topology and location of the required stabilization network. An admittance plot has allowed a quick test for global stabilization. With the inclusion of the stabilization network, the PA behaves in a globally stable manner with minimum degradation in drain efficiency and output power.

\section{ACKNOWLEDGMENT}

The authors would like to thank S. Weinreb, Jet Propulsion Laboratory, Pasadena, CA, K. Potter and F. Wang, both of the California Institute of Technology, Pasadena, and J.-M. Collantes, University of the Basque Country, Bilbao, Spain, for their advice and discussions.

\section{REFERENCES}

[1] H. L. Krauss, C. W. Bostian, and F. H. Raab, Solid State Radio Engineering. New York: Wiley, 1980.

[2] S. D. Kee, I. Aoki, A. Hajimiri, and D. B. Rutledge, "The class-E/F family of ZVS switching amplifiers," IEEE Trans. Microw. Theory Tech., vol. 51, no. 6, pp. 1677-1690, Jun. 2003.

[3] E. Lau, K.-W. Chiu, J. Qin, J. Davis, K. Potter, and D. Rutledge, "High-efficiency class-E power amplifiers-Part 1," $Q S T$, pp. 39-42, May 1997.

[4] M. Albulet, RF Power Amplifiers. New York: Noble, 2001.

[5] J. Vidkjaer, "Instabilities in RF-power amplifiers caused by a self-oscillation in the transistor bias network," IEEE J. Solid-State Circuits, vol. SSC-11, no. 10, pp. 703-712, Oct. 1976.

[6] A. Anakabe, J. M. Collantes, J. Portilla, J. Jugo, S. Mons, A. Mallet, and L. Lapierre, "Analysis of odd-mode parametric oscillations in HBT multi-stage power amplifiers," in Eur. Microwave Week-11th GaAs Symp., Munich, Germany, Oct. 2003, pp. 533-536.

[7] A. Collado, F. Ramírez, and A. Suárez, "Analysis and stabilization tools for microwave amplifiers," in IEEE MTT-S Int. Microwave Symp. Dig., Fort Worth, TX, Jun. 2004, pp. 945-948.

[8] S. Mons, J.-C. Nallatamby, R. Queré, P. Savary, and J. Obregon, “A unified approach for the linear and nonlinear stability analysis of microwave circuits using commercially available tools," IEEE Trans. Microw. Theory Tech., vol. 47, no. 12, pp. 2403-2409, Dec. 1999.

[9] J. M. T. Thompson and H. B. Stewart, Nonlinear Dynamics and Chaos. New York: Wiley, 1986.

[10] D. Teeter, A. Platzker, and R. Bourque, "A compact network for eliminating parametric oscillations in high power MMIC amplifiers," in IEEE MTT-S Int. Microwave Symp. Dig., Anaheim, CA, Jun. 1999, pp. 967-970. 
[11] M. Mochizuki, M. Nakayama, Y. Tarui, Y. Itoh, S. Tsuji, and T. Takagi, "Nonlinear analysis of $f_{o} / 2$ loop oscillation of high power amplifiers," in IEEE MTT-S Int. Microwave Symp. Dig., Orlando, FL, May 1995, pp. 709-712.

[12] I. Aoki, S. D. Kee, D. B. Rutledge, and A. Hajimiri, "Distributed active transformer-A new power-combining and impedance-transformation technique," IEEE Trans. Microw. Theory Tech., vol. 50, no. 1, pp. 316-331, Jan. 2002.

[13] S. Jeon and D. B. Rutledge, "A 2.7-kW, 29-MHz class-E/F $F_{\text {odd }}$ amplifier with a distributed active transformer," presented at the IEEE MTT-S Int. Microwave Symp., Long Beach, CA, Jun. 2005.

[14] Adv. Power Technol. Inc., Bend, OR, VDMOS Data Sheet for ARF473, 2003. [Online]. Available: http://www.advancedpower.com.

[15] "Spice model for TMOS power MOSFETs," Motorola Semiconduct. Products Inc., Phoenix, AZ, Applicat. Note AN1043, 1989.

[16] Y.-S. Kim and J. G. Fossum, "Physical DMOST modeling for highvoltage IC CAD," IEEE Trans. Electron Devices, vol. 37, no. 3, pp. 797-803, Mar. 1990.

[17] V. Iglesias, A. Suárez, and J. L. García, "New technique for the determination through commercial software of the stable-operation parameter ranges in nonlinear microwave circuits," IEEE Microw. Guided Wave Lett., vol. 8, no. 12, pp. 424-426, Dec. 1998.

[18] J. Jugo, J. Portilla, A. Anakabe, A. Suárez, and J. M. Collantes, "Closedloop stability analysis of microwave amplifiers," Electron. Lett., vol. 37, pp. $226-228$, Feb. 2001.

[19] K. Kurokawa, "Some basic characteristics of broad-band negative resistance oscillator circuits," Bell Syst. Tech. J., vol. 48, pp. 1937-1955, Jul.-Aug. 1969.

[20] J. Jugo, A. Anakabe, and J. M. Collantes, "Control design in the harmonic domain for microwave and RF circuits," Proc. Inst. Elect. Eng., Contr. Theory Applicat., vol. 150, no. 2, pp. 127-131, Mar. 2003.

[21] A. Suárez and R. Queré, Global Stability Analysis of Microwave Circuits. Boston, MA: Artech House, 2003.

[22] J. Guckenheimer and P. Holmes, Nonlinear Oscillations, Dynamical Systems and Bifurcations of Vector Fields, 3rd ed. Berlin, Germany: Springer-Verlag, 1990.

[23] E. Ngoya and R. Larcheveque, "Envelope transient analysis: A new method for the transient and steady state analysis of microwave communication circuits and systems," in IEEE MTT-S Int. Microwave Symp. Dig., San Francisco, CA, Jun. 1996, pp. 1365-1368.

[24] J. C. Pedro and N. B. Carvalho, "Simulation of RF circuits driven by modulated signals without bandwidth constraints," in IEEE MTT-S Int. Microwave Symp. Dig., Seattle, WA, Jun. 2002, pp. 2173-2176.

[25] E. de Cos, A. Suárez, and S. Sancho, "Envelope transient analysis of self-oscillating mixers," IEEE Trans. Microw. Theory Tech., vol. 52, no. 4, pp. 1090-1100, Apr. 2004.

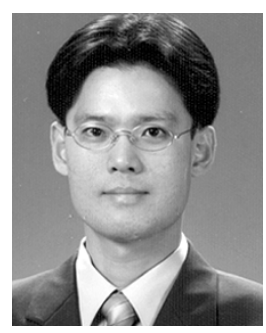

Sanggeun Jeon (S'05) received the B.S. and M.S. degrees in electrical engineering from Seoul National University, Seoul, Korea, in 1997 and 1999, respectively, the M.S. degree in electrical engineering from the California Institute of Technology, Pasadena, in 2004, and is currently working toward the Ph.D. degree at the California Institute of Technology.

From 1999 to 2002, he was a Full-Time Instructor of electronics engineering with the Korea Air Force Academy, Cheongwon, Korea. His research interests include high-efficiency PAs and nonlinear stability analysis.

Mr. Jeon was the recipient of the Third Place Award in the Student Paper Competition at the 2005 IEEE Microwave Theory and Techniques Society (IEEE MTT-S) International Microwave Symposium (IMS).

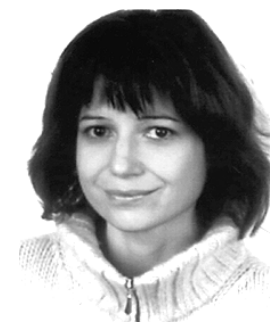

Almudena Suárez (M'96-SM'01) was born in Santander, Spain. She received the Electronic Physics and Ph.D. degrees from the University of Cantabria, Santander, Spain, in 1987 and 1992, respectively, and the Ph.D. degree in electronics from the University of Limoges, Limoges, France, in 1993.

In 1987, she joined the Electronics Department, University of Cantabria, where she was involved with nonlinear simulation. From May 1990 to December 1992, she was on leave with the Institute de Recherche en Communications Optiques et Microondes (IRCOM), University of Limoges. Since 1993, she has been an Associate Professor (permanent since June 1995) with the Communications Engineering Department, University of Cantabria. She coauthored Stability Analysis of Microwave Circuits (Norwood, MA: Artech House, 2003). Her areas of interest include the nonlinear design of microwave circuits, especially the nonlinear stability and phase-noise analysis and the investigation of chaotic regimes.

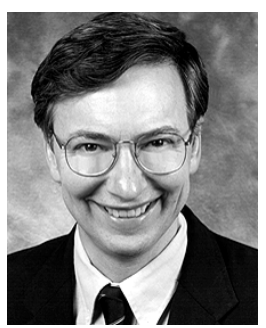

David B. Rutledge (S'77-M'77-SM'89-F'93) received the B.A. degree in mathematics from Williams College, Williamstown, MA, the M.A. degree in electrical sciences from Cambridge University, Cambridge, U.K., and the Ph.D. degree in electrical engineering from the University of California at Berkeley.

He is currently the Tomiyasu Professor of Electrical Engineering with the California Institute of Technology, Pasadena. He is Director of the California Institute of Technology's Lee Center for Advanced Networking. He authored the electronics textbook The Electronics of Radio (Cambridge, U.K.: Cambridge Univ. Press, 1999) and coauthored the microwave computer-aided-design software package Puff, which has sold 30000 copies. His research has been in integrated-circuit antennas, active quasi-optics, computer-aided design, and high-efficiency PAs.

Prof. Rutledge was the recipient of the Microwave Prize, the Distinguished Educator Award of the IEEE Microwave Theory and Techniques Society (IEEE MTT-S), the Teaching Award of the Associated Students of the California Institute of Technology, the Doug DeMaw Award of the American Radio Relay League (ARRL), and the Third Millennium Award of the IEEE. He was the editor-in-chief of the IEEE TRANSACTIONS ON MicRowAVE THEORY AND TEChNIQUes, and a Distinguished Lecturer of the IEEE Antennas and Propagation Society (IEEE AP-S). 